\title{
COX-2 Expression in the Aorta of Obese Zucker Rats
}

Tomoko Shimomura $^{1 \#}$, Tomoyuki Nakano ${ }^{2 \#}$, Kaoru Goto ${ }^{2}$ and Ichiro Wakabayashi ${ }^{1 *}$

${ }^{1}$ Department of Environmental and Preventive Medicine, Hyogo College of Medicine, Nishinomiya, Hyogo, Japan

${ }^{2}$ Department of Anatomy and Cell Biology, Yamagata University Faculty of Medicine, Yamagata, Japan

"Corresponding author: Ichiro Wakabayashi, Department of Environmental and Preventive Medicine, Hyogo College of Medicine, Mukogawa-cho 1-1, Nishinomiya, Hyogo, Tel: +81-798-45-6561; E-mail: wakabaya@hyo-med.ac.jp

\#Both contributed equally to this work.

Received date: February 28, 2018; Accepted date: March 15, 2018; Published date: March 19, 2018

Copyright: @ 2018 Shimomura T, et al. This is an open-access article distributed under the terms of the creative commons attribution license, which permits unrestricted use, distribution, and reproduction in any medium, provided the original author and source are credited.

\begin{abstract}
Objectives: Obesity is a central risk factor for atherosclerotic cardiovascular disease. The purpose of this study was to determine whether COX-2 expression is changed in the arterial wall of experimental obese animals.

Methods: COX-2 expression in the aortas of 12-week-old male Zucker obese or lean rats was investigated using immunoblot and immunohistochemical analyses.

Results: COX-2 expression was detected in the tunica media of the aortas of Zucker obese rats and was prominent in the perinuclear region of smooth muscle cells in the tunica media. In immunoblot analysis, the basal (non-stimulated) levels of COX-2 expression were comparable in the aortas of Zucker obese rats and Zucker lean (control) rats. COX-2 expression in response to interleukin (IL)-1 $\beta$ was significantly lower in the aortas of Zucker obese rats than in those of Zucker lean rats.
\end{abstract}

Conclusions: The results suggest that IL-1 $\beta$-induced COX-2 expression is attenuated in arteries of obese rats, and this might be involved in the obesity-induced acceleration of atherosclerosis.

Keywords: Arterial smooth muscle cells; Atherosclerosis; COX-2; Obesity; Zucker rats

\section{Introduction}

Effects of obesity, a central risk factor for cardiovascular disease, such as coronary heart disease and stroke, are mediated through the risks of metabolic syndrome including insulin resistance, dyslipidemia and hypertension [1]. Atherosclerosis, which is the basic pathogenesis of cardiovascular disease, is accelerated by metabolic syndrome [2]. Atherosclerosis is characterized by a persistent low-grade inflammatory state [3], which is also presented by obesity status [4].

Cyclooxygenase (COX) is the rate-limiting enzyme catalyzing conversion of arachidonic acid to prostaglandins, which include major inflammatory mediators. However, the pathophysiological role of COX-2, an inducible isoform of COX, in atherothrombosis in cardiovascular disease remains to be clarified [5-7]. Among the prostanoids synthesized through the COX-2-mediated pathway, prostaglandin E2 and prostacyclin are deeply involved in the pathogenesis of atherosclerosis. In atherosclerotic lesions, the former plays a role in inflammatory processes, while the latter inhibits thrombosis and ischemia. Thus, prostaglandin E2 and prostacyclin are thought to be the key prostanoids in cardiovascular homeostasis [8]. There have been few studies on the relationship between obesity and COX-2 expression in blood vessels. The obese Zucker rat (OZR) is a spontaneous genetic obesity model, in contrast to the lean Zucker rat (LZR) [9]. COX-2 expression in the coronary artery has recently been shown to be higher in OZRs than in LZRs [10]. The purpose of this study was to determine whether COX-2 expression is altered in aortas of OZRs. COX-2 expression was evaluated in the absence and presence of interleukin (IL)-1 $\beta$, a major proinflammatory cytokine.

\section{Materials and Methods}

\section{Experimental animals and preparation of aortic tissues}

Aortic ring-shaped strips (approximately $5 \mathrm{~mm}$ long) were prepared under sterile conditions from 12-week-old male OZRs or LZRs (Charles River Inc.) $(n=5)$ as described previously [11]. We used only male rats in this study because results of experiments using female animals are considerably influenced by their hormonal condition. In fact, prostacyclin synthesis is known to be increased by estrogen [12]. The study protocols regarding treatment of rats were in accordance with the Guidelines for Experiments Using Laboratory Animals in Hyogo College of Medicine. Each strip was put in a well of a 24-well plate containing $0.5 \mathrm{ml}$ of Dulbecco's Modified Eagle's medium (DMEM) with $4 \mathrm{mM}$ glutamate, $100 \mathrm{U} / \mathrm{ml}$ penicillin, $100 \mu \mathrm{g} / \mathrm{ml}$ of streptomycin and $0.25 \mu \mathrm{g} / \mathrm{ml}$ of amphotericin B. The plates were incubated in a humidified atmosphere at $37^{\circ} \mathrm{C}$ under $5 \% \mathrm{CO}_{2}-95 \%$ air. After being stabilized in DMEM for 3 hours, each strip was incubated for 24 hours in the presence of interleukin $1 \beta$ (IL-1 $\beta$ ) at $10 \mathrm{ng} / \mathrm{ml}$ or a vehicle (phosphate-buffered saline). The aortic strips were then used for experiments. Body weight and weight of epididymal white adipose tissues (eWATs) were measured.

\section{Histological analysis of aortas, adipose tissues and livers}

Aortic strips were fixed with $4 \%$ paraformaldehyde/0.1 M phosphate buffer ( $\mathrm{pH}$ 7.4). For adipose tissue analysis, eWATs were dehydrated 
through a graded ethanol series and were embedded in paraffin. Six$\mu \mathrm{m}$ thick sections were cut and stained by hematoxylin and eosin. For liver tissue examination, fixed tissues were immersed in $30 \%$ sucrose for cryoprotection and were cut into $12-\mu \mathrm{m}$ thick frozen sections on a cryo-stat. Frozen sections were stained with Oil-Red-O.

\section{Immunohistochemical analysis of aortic tissues}

Frozen sections were treated with $0.3 \%$ Triton X-100/PBS followed by $0.3 \% \mathrm{H}_{2} \mathrm{O}_{2}$, and then nonspecific binding sites were blocked with $5 \%$ normal goat serum. The sections were reacted with anti-rat COX-2 antibody (Cayman Chemical, Ann Arbor, MI, USA) in a moisture chamber overnight. Then the sections were reacted with biotinylated anti-rabbit IgG (Vector Laboratories, Burlingame, CA, USA). The immunoreactions were detected using an $\mathrm{ABC}$ kit (Vector Laboratories) and diaminobentidine.

\section{Immunoblot analysis of aortic tissues}

Aortic strips were homogenized in RIPA buffer containing $1 \%$ NP- $40,0.5 \%$ sodium deoxycholate, $0.1 \%$ SDS, a complete protease inhibitor tablet (Roche Applied Science, Indianapolis, IN, USA), and 1 $\mathrm{mM} \mathrm{NaVO}_{4}$ and $1 \mathrm{mM}$ PMSF by using a small plastic pestle followed by sonication. After centrifugation at $12000 \times \mathrm{xg}$ for $10 \mathrm{~min}$ at $4^{\circ} \mathrm{C}$, the supernatant was used to measure protein concentration, and each of the protein samples was subjected to SDS/PAGE. Antibodies against COX-2 or a-tubulin (Cell Signaling Technology, Beverly, MA, USA) were used after diluting 1:1000 with $2 \mathrm{ml}$ of TBS containing $0.1 \%$ Tween 20 in each experiment of immunoblotting. Immunoreactions for COX-2 and $\alpha$-tubulin antibodies were visualized using an Immobilon Western (Millipore, Bedford, MA, USA)

\section{Statistical analysis}

Each variable was compared between OZRs and LZRs using Student's $t$ test or one-way ANOVA followed by the Scheffé's test. Probability (p) values less than 0.05 were defined as significant

\section{Results}

Table 1 shows characteristics of OZRs and LZRs. Body weight, eWAT weight and the ratio of eWAT weight to body weight were significantly larger in OZRs than in LZRs. Figure 1 shows hematoxylin \& eosin and oil-red staining images of eWAT and the liver, respectively. Adipocytes of OZRs were larger than those of LZRs. Prominent fat accumulation and hepatocellular ballooning were observed in the liver of OZRs but not in the liver of LZRs.

\begin{tabular}{|l|l|l|}
\hline Group & BW $(\mathbf{g})$ & eWAT weight $\mathbf{( g )}$ \\
\hline Lean & $335.0 \pm 9.4$ & $4.10 \pm 0.26$ \\
\hline Obese & $459.8 \pm 8.1^{* *}$ & $12.6 \pm 0.3^{* *}$ \\
\hline
\end{tabular}

Means with standard errors of each variable are shown. BW, body weight; eWAT, epididymal white adipose tissue. Asterisks denote significant differences from Zucker lean rats $\left({ }^{* *} p<0.01\right)$.

Table 1: Characteristics of Zucker lean and obese rats.

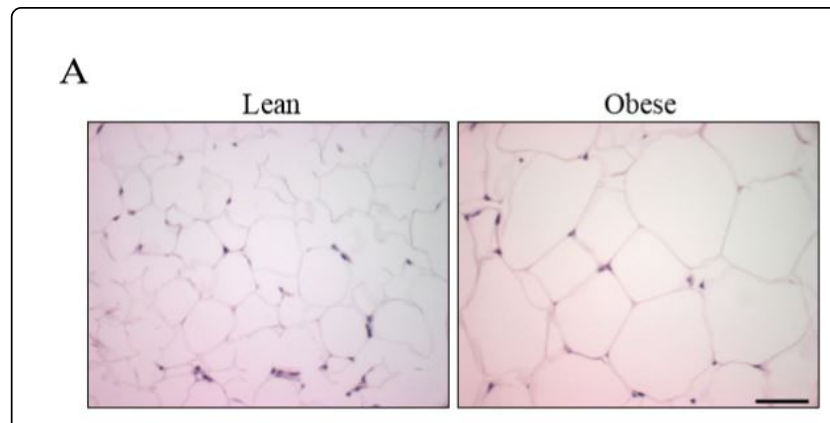

B

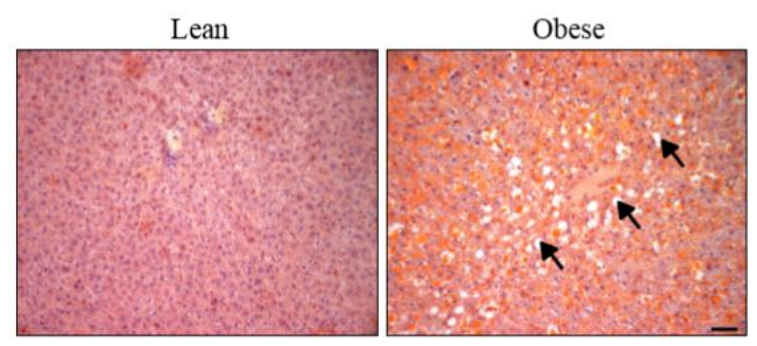

Figure 1: Histological analysis of epididymal white adipose tissue (A) and liver (B) from Zucker rats. H\&E staining revealed a larger adipocyte size in OZRs than in LZRs. Bar $=50 \mu \mathrm{m}$ (A). Oil red O staining demonstrated that severe hepatic steatosis and ballooning were induced in the OZR liver. Nuclear staining was performed by hematoxylin. Arrows indicate hepatic ballooning. Bar=50 $\mu \mathrm{m}$ (B).

Figure 2 shows immunostaining images of COX-2 in aortic tissues of OZRs. Immunoreactivity for COX-2 antibody was detected in the tunica media, but no immnoreactivity of COX-2 was detected in the tunica intima and adventitia of the aortic wall. Confocal microscopic observation of aortic tissues with DAPI nuclear staining showed COX-2-immunoreactivity in the perinuclear region of medial cells.

Figure 3 shows images of immunoblot of COX-2 in aortic tissues of OZRs and LZRs and the results of its densitometric analysis. Weak expression of COX-2 was detected in the absence of IL- $1 \beta$, and stimulation of the aortic tissues with IL- $1 \beta$ resulted in an increase of COX-2 expression (Figure 3A). Densitometric analysis of COX-2specific density corrected by a-tubulin-specific density of each aortic strip revealed that COX-2 expression in aortas at the basal level without IL- $1 \beta$ stimulation was not significantly different in OZRs and LZRs. COX-2 expression levels in aortae after IL- $1 \beta$ stimulation tended to be lower in OZRs than in LZRs, although the ratios of COX-2specific density to tubulin-specific density were not significantly different in OZRs and LZRs (Figure 3B). The ratio of COX-2 expression in the presence of IL- $1 \beta$ to that in its absence (fold increase of COX- 2 expression by IL- $1 \beta$ stimulation) was significantly lower in OZRs than in LZRs (Figure 3C). 
Page 3 of 4

A

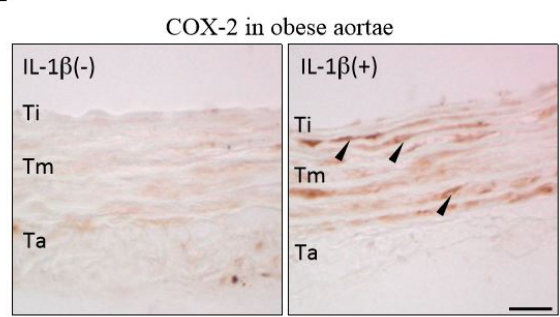

B
COX-2/IL-1 $\beta$

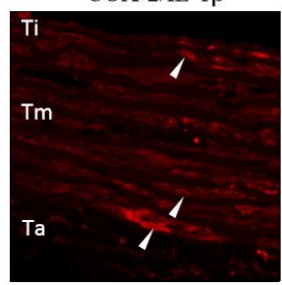

merged with DAPI

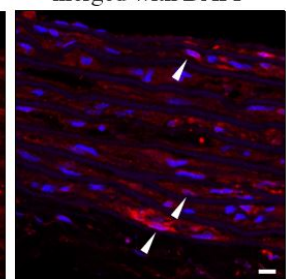

Figure 2: Representative images of COX-2 immunohistochemistry of the OZR aorta in the presence (right image) and absence (left image) of IL- $1 \beta$ stimulation (A) and confocal micrographs of the OZR aorta in the presence of IL-1 $\beta$ stimulation (B). A: Intense immunoreactivity for COX-2 antibody was detected in the tunica media of IL- $\beta$-stimulated aortic rings (arrowheads). Bar $=25 \mu \mathrm{m}$. B: Immunoreactions for COX-2 were detected in the perinuclear area of cells in the tunica media (arrowheads). Nuclear staining was performed with DAPI. Bar $=10 \mu \mathrm{m}$. Ti, tunica intima; Tm, tunica media; Ta, tunica adventitia.

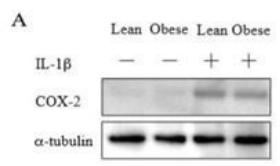

B
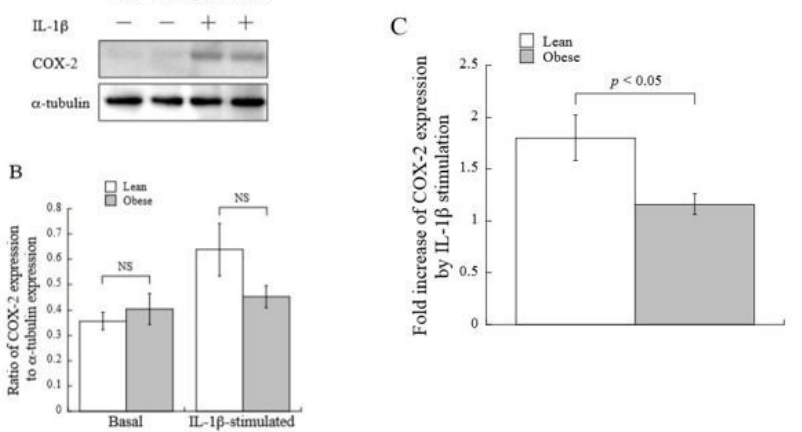

Figure 3: Immunoblot analysis of COX-2 in aortic rings of Zucker rats in the presence and absence of IL- $1 \beta$ stimulation. A: Representative images of COX-2 imunoblot analysis. Aortic rings were incubated with IL-1 $\beta(10 \mathrm{ng} / \mathrm{ml})$ for $24 \mathrm{hr}$. $\alpha$-Tubulin was used as a control. B and C: Densitometric analysis of COX-2 protein expression. Density of the band for COX-2 was corrected by that of $a$-tubulin. Data are shown as means \pm standard errors. $n=5$. NS, no significant difference.

\section{Discussion}

This study demonstrated for the first time that COX-2 is expressed in the tunica media of the aorta of OZRs and that IL- $1 \beta$-stimulated COX-2 expression is attenuated in OZRs, while the basal expression levels of COX-2 are not different in OZRs and LZRs. Regarding loci of COX-2 expression in the aortic wall, COX-2 expression was detected only in the tunica media of aortas from OZRs in the presence or absence of IL-1 $\beta$. However, in our previous study using aortas of normal Wistar rats, COX-2 expression was immunohistochemically detected in the tunica adventica but not in the tunica media or intima [13]. In a recent study using coronary arteries of OZRs and LZRs, COX-2 expression was detected in the endothelium and vascular smooth muscle [10]. Immunoreactivity for COX-2 was found in the endothelium of renal arteries of LZRs and in both the endothelium and smooth muscle of renal arteries of OZRs [14]. COX-2 was reported to be expressed in the endothelium of penile arteries from both LZRs and OZRs [15]. Thus, the distribution of COX expression in arteries may differ depending on the species of rat and the loci of artery.

Although the pathophysiological significance of COX-2 in cardiovascular disease remains obscure [5-7], it has been pointed out that prostacyclin produced through the COX-2 pathway plays a protective role in progression of atherosclerosis since prostacyclin has anti-thrombotic and vasodilatory actions. Cytokines including IL-1 $\beta$ are deeply involved in inflammatory responses in atherosclerotic vessels [16]. In the present study, COX-2 expression was found to be decreased in OZRs rats compared to that in LZRs. Thus, a decrease in COX-2 expression in arterial smooth muscle cells might contribute to acceleration of atherosclerotic progression in an obesity state, a major risk factor of cardiovascular disease. To the best of our knowledge, there has been no report on a comparison of COX-2 expression levels in the aortas of obese and control rats. A recent study by Santiago et al. showed that the basal level of COX-2 expression in the coronary artery was significantly higher in OZRs than in LZRs, while levels of COX-2 expression stimulated by $\mathrm{H}_{2} \mathrm{O}_{2}$ were comparable in the two groups of Zucker rats [10]. Thus, the results of a comparison of basal COX-2 expression levels were different in the previous study by Santiago et al. using coronary arteries and in the present study using aortas. In coronary arteries of LZRs, stimulation with $\mathrm{H}_{2} \mathrm{O}_{2}$ resulted in an increase of COX-2 expression, while no increase of COX-2 in response to $\mathrm{H}_{2} \mathrm{O}_{2}$ was found in coronary arteries of OZRs [10]. This agrees with the finding in the present study that the increase of COX-2 expression in response to IL- $1 \beta$ was attenuated in aortas of OZRs compared with that in aortas of LZRs.

There are limitations of this study. OZR is a representative animal model of obesity, and further studies are needed to elucidate the localization of and change in COX-2 expression in arteries of obese humans. Measurement of the levels of COX-2-mediated production of prostaglandins is needed to elucidate the pathophysiological significance of the change in COX-2 expression in aortas of OZRs. Further studies with experiments on upper-stream signal transduction and gene expression of COX-2 are also needed to clarify the mechanism for attenuation of IL- $1 \beta$-stimulated COX- 2 expression in arteries of OZRs.

In conclusion, COX-2 is expressed in aortic smooth muscle of OZRs, and IL- $1 \beta$-induced COX- 2 expression is attenuated in OZRs compared with that in LZRs. Attenuation of COX-2 expression in arterial smooth muscle might be involved in the obesity-induced acceleration of atherosclerotic progression. 
Citation: Shimomura T, Nakano T, Goto K, Wakabayashi I (2018) COX-2 Expression in the Aorta of Obese Zucker Rats. J Metabolic Synd 7: 238. doi:10.4172/2167-0943.1000238

Page 4 of 4

\section{Acknowledgements}

This work was supported by a Grant-in-Aid for researchers at Hyogo College of Medicine (2014) (to Tomoko Shimomura).

\section{References}

1. The Global Burden of Metabolic Risk Factors for Chronic Diseases Collaboration (BMI Mediated Effects (2014) Metabolic mediators of the effects of body-mass index, overweight, and obesity on coronary heart disease and stroke: a pooled analysis of 97 prospective cohorts with 1.8 million participants. Lancet 383: 970-983.

2. Malik S, Wong ND (2009) Metabolic syndrome, cardiovascular risk and screening for subclinical atherosclerosis. Expert Rev Cardiovasc Ther 7: 273-280.

3. Tsirpanlis G (2005) Inflammation in atherosclerosis and other conditions: a response to danger. Kidney Blood Press Res 28: 211-217.

4. Ruscica M, Baragetti A, Catapano AL, Norata GD (2017) Translating the biology of adipokines in atherosclerosis and cardiovascular diseases: Gaps and open questions. Nutr Metab Cardiovasc Dis 27: 379-395.

5. Cuccurullo C, Fazia ML, Mezzetti A, Cipollone F (2007) COX-2 expression in atherosclerosis: the good, the bad or the ugly? Curr Med Chem 14: 1595-1605.

6. Cuccurullo C, Mezzetti A, Cipollone F (2007) COX-2 and the vasculature: angel or evil? Curr Hypertens Rep 9: 73-80.

7. Iezzi A, Ferri C, Mezzetti A, Cipollone F (2007) COX-2: friend or foe? Curr Pharm Des 13: 1715-1721.

8. Kurtz TW, Morris RC, Pershadsingh HA (1989) The Zucker fatty rat as a genetic model of obesity and hypertension. Hypertension 13: 896-901.
9. Funk CD, FitzGerald GA (2007) COX-2 inhibitors and cardiovascular risk. J Cardiovasc Pharmacol 50: 470-479.

10. Santiago E, Martínez MP, Climent B, Muñoz M, Briones AM, et al. (2016) Augmented oxidative stress and preserved vasoconstriction induced by hydrogen peroxide in coronary arteries in obesity: role of COX-2. Br J Pharmacol 173: 3176-3195.

11. Takahashi Y, Wakabayashi I (2008) Depression of cyclooxygenase-2 induction in aortas of rats with type 1 and type 2 diabetes mellitus. Eur J Pharmacol 595: 114-118.

12. Mikkola T, Viinikka L, Ylikorkala O (1998) Estrogen and postmenopausal estrogen/progestin therapy: effect on endothelium-dependent prostacyclin, nitric oxide and endothelin-1 production. Eur J Obstet Gynecol Reprod Biol 79: 75-82.

13. Nakano T, Wakabayashi I (2010) Identification of cells expressing cyclooxygenase- 2 in response to interleukin- $1 \beta$ in rat aortae. J Pharmacol Sci 113: 84-88.

14. Muñoz M, Sánchez A, Pilar Martínez M, Benedito S, López-Oliva ME, et al. (2015) COX-2 is involved in vascular oxidative stress and endothelial dysfunction of renal interlobar arteries from obese Zucker rats. Free Radic Biol Med 84: 77-90.

15. Sánchez A, Contreras C, Villalba N, Martínez P, Martínez AC, et al. (2010) Altered arachidonic acid metabolism via COX-1 and COX-2 contributes to the endothelial dysfunction of penile arteries from obese Zucker rats. Br J Pharmacol 159: 604-616.

16. Warnatsch A, Ioannou M, Wang Q, Papayannopoulos V (2015) Neutrophil extracellular traps license macrophages for cytokine production in atherosclerosis. Science 349: 316-20. 